The capacity region of the restricted two-way relay channel with any deterministic uplink

by Lawrence Ong \& Sarah J. Johnson

Copyright (C) 2012 IEEE.

This is an author-prepared version of the article, reprinted from IEEE Communications Letters Vol. 16, Issue 3, p. 396-399.

http://dx.doi.org/10.1109/LCOMM.2012.011312.112198

This material is posted here with permission of the IEEE. Such permission of the IEEE does not in any way imply IEEE endorsement of any of University of Newcastle's products or services. Internal or personal use of this material is permitted. However, permission to reprint/republish this material for advertising or promotional purposes or for creating new collective works for resale or redistribution must be obtained from the IEEE by writing to pubs-permissions@ieee.org. By choosing to view this document, you agree to all provisions of the copyright laws protecting it. 


\title{
The Capacity Region of the Restricted Two-Way Relay Channel with Any Deterministic Uplink
}

\author{
Lawrence Ong and Sarah J. Johnson
}

\begin{abstract}
This paper considers the two-way relay channel (TWRC) where two users communicate via a relay. For the restricted TWRC where the uplink from the users to the relay is any deterministic function and the downlink from the relay to the users is any arbitrary channel, the capacity region is obtained. The TWRC considered is restricted in the sense that each user can only transmit a function of its message.
\end{abstract}

\section{INTRODUCTION}

The two-way relay channel (TWRC), where two users exchange data through a relay, was first investigated by $\mathrm{Wu}$ et al. [1]. In this model the users cannot communicate directly and must exchange data by first transmitting to the relay, called the uplink. The relay then processes the data in some way and broadcasts to both users on the downlink. Common applications that can be modeled by the TWRC include satellite communications, cellular communications via a base station, and indoor wireless communications via a router. Using relays to facilitate data exchange is now moving from theory to practice following their introduction in the 802.16j (WiMAX) standard.

The capacity region of the two-way relay channel was found for the case where the uplink and the downlink are both binary symmetric adder channels [2]. Since then the capacity region of more general TWRCs has been found for only a few classes of channel models: (i) the uplink and the downlink are both finite-field adder channels [3], and (ii) the uplink and the downlink are both linear finite-field deterministic channels [4]. For the Gaussian TWRC, results within $\frac{1}{2}$ bit of the capacity have been obtained [5].

In all the classes of TWRCs where the capacity is known, the uplink channels are linear. In this paper, we derive the capacity region of another class of TWRC, where (i) the uplink is any deterministic channel ${ }^{1}$, (ii) the downlink is any arbitrary channel $^{2}$, and (iii) the users' channel inputs can only depend on their respective messages, and not on their received channel outputs (this is commonly known as the restricted channel). To the best of our knowledge, this is the first class of TWRCs with non-linear uplinks where the capacity region is found.

Deterministic channels can model networks comprising fixed-capacity links, and can approximate channels with extremely low noise. Another advantage of the deterministic approach is that one can focus on the interaction between

This work is supported by the Australian Research Council under the grant DP1093114.

${ }^{1}$ This includes the linear finite-field deterministic model as a special case but also includes non-linear channels.

${ }^{2}$ This includes the finite-field adder and Gaussian channels as special cases.

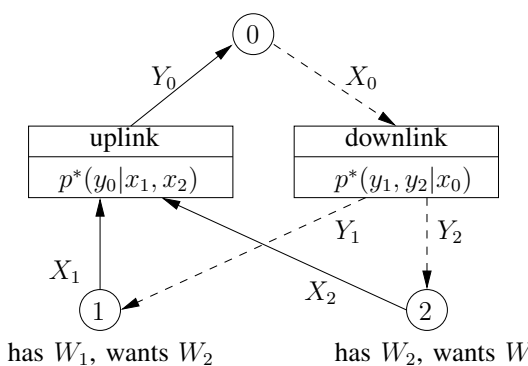

Fig. 1. The two-way relay channel. For a deterministic uplink, $Y_{0}=$ $f\left(X_{1}, X_{2}\right)$ is a deterministic function.

the signals arriving from different nodes rather than the background noise of the system [4].

We will show that the capacity region of the restricted TWRC with a deterministic uplink and an arbitrary downlink can be achieved using the compress-forward (CF) coding scheme. This scheme was first proposed by Cover and El Gamal for the single-source single-destination single-relay channel [6]. Using CF the relay does not decode the received signals but rather compresses them (using Wyner-Ziv coding), bins the compressed signals (using the random-binning technique), and sends the bin index. CF was extended to the TWRC by Rankov and Wittneben [7] and Schnurr et al. [8].

In this work, we obtain the capacity of the restricted deterministic-uplink arbitrary-downlink TWRC in Section IV by showing that the capacity outer bound derived using the cut-set argument [9] coincides with the capacity inner bound derived using $\mathrm{CF}$ [8]. In the absence of noise on the uplink, we can set the "quantization noise" of $\mathrm{CF}$ to zero, i.e., having the relay directly map its received signals to its transmitted signals. In this case, binning is also not required. In the light of this observation, we present an alternative proof of the capacity region in Section V using a simpler coding scheme.

\section{Channel Model}

The TWRC (see Fig. 1) consists of three nodes: two users (denoted by nodes 1 and 2) and one relay (denoted by node $0)$. Let $X_{i}$ be the channel input from node $i$ and $Y_{i}$ be the channel output received by node $i$. The general (not necessarily deterministic) TWRC is defined as $p\left(y_{0}, y_{1}, y_{2} \mid x_{0}, x_{1}, x_{2}\right)=$ $p^{*}\left(y_{0} \mid x_{1}, x_{2}\right) p^{*}\left(y_{1}, y_{2} \mid x_{0}\right)$.

We consider $n$ channel uses, and denote the channel variables $X_{i}$ and $Y_{i}$ at time $t$ as $X_{i, t}$ and $Y_{i, t}$ respective, for $t \in$ $\{1,2, \ldots, n\}$. Our block codes consists of the following: (i) An independent message for each user $i, W_{i} \in\left\{1,2, \ldots, 2^{n R_{i}}\right\}$ for $i \in\{1,2\}$; (ii) Encoding functions for the users, $X_{i, t}=$ $g_{i, t}\left(W_{i}\right)$ for $i \in\{1,2\}$ and $t \in\{1, \ldots, n\}$; (iii) Encoding 
functions for the relay, $X_{0, t}=g_{0, t}\left(Y_{0,1}, Y_{0,2}, \ldots, Y_{0, t-1}\right)$ for $t \in\{1, \ldots, n\}$; and (iv) A decoding function for each user, $\hat{W}_{j}=h_{i}\left(W_{i}, Y_{i, 1}, Y_{i, 2}, \ldots, Y_{i, n}\right)$. Here $\hat{W}_{j}$ is the estimate of the message $W_{j}$ by user $i, i \neq j$. Note that in each channel usage, each user transmits a function of its own message (i.e., it is a restricted channel), and the relay transmits a function of its previously received channel outputs.

We say that the rate pair $\left(R_{1}, R_{2}\right)$ is achievable if the following is true: for any $\epsilon>0$, there exists at least one block code such that $\operatorname{Pr}\left\{\hat{W}_{1} \neq W_{1}\right.$ or $\left.\hat{W}_{2} \neq W_{2}\right\} \leq \epsilon$. The capacity region $\mathcal{C}$ is the closure of all achievable rate pairs.

\section{CAPACITY OUTER BOUND AND INNER BOUND}

We review an outer bound and an inner bound to $\mathcal{C}$. Let

$$
\begin{aligned}
& \mathcal{R}_{1} \triangleq\left\{\left(R_{1}, R_{2}\right)\right. \in \mathbb{R}_{+}^{2}: \\
& R_{1} \leq I\left(X_{1} ; Y_{0} \mid X_{2}\right) \\
& R_{2} \leq I\left(X_{2} ; Y_{0} \mid X_{1}\right),
\end{aligned}
$$

for some $\left.p\left(x_{1}, x_{2}, y_{0}\right)=p\left(x_{1}\right) p\left(x_{2}\right) p^{*}\left(y_{0} \mid x_{1}, x_{2}\right)\right\}$,

$$
\begin{array}{r}
\mathcal{R}_{2} \triangleq\left\{\left(R_{1}, R_{2}\right) \in \mathbb{R}_{+}^{2}:\right. \\
R_{1} \leq I\left(X_{0} ; Y_{2}\right) \\
R_{2} \leq I\left(X_{0} ; Y_{1}\right),
\end{array}
$$

for some $\left.p\left(x_{0}, y_{1}, y_{2}\right)=p\left(x_{0}\right) p^{*}\left(y_{1}, y_{2} \mid x_{0}\right)\right\}$.

Denote the convex hull of a set $\mathcal{R}$ as $\operatorname{Conv}(\mathcal{R})$, and define

$$
\mathcal{R}_{\text {out }} \triangleq \operatorname{Conv}\left(\mathcal{R}_{1}\right) \cap \mathcal{R}_{2} \text {. }
$$

Remark 1: The set $\operatorname{Conv}\left(\mathcal{R}_{1}\right)$ is closed [10, pg. 625], and the set $\mathcal{R}_{2}$ is convex and closed [9].

The following outer bound is due to Fong and Yeung [9]:

Lemma 1: $\mathcal{C} \subseteq \mathcal{R}_{\text {out }}$.

Using the $\mathrm{CF}$ coding scheme, the following rate region is achievable (i.e., an inner bound to the capacity) [8]:

Lemma 2: $\mathcal{R}_{\mathrm{CF}} \subseteq \mathcal{C}$, where

$$
\begin{aligned}
\mathcal{R}_{\mathrm{CF}} \triangleq\{( & \left.R_{1}, R_{2}\right) \in \mathbb{R}_{+}^{2}: \\
& R_{1}<I\left(X_{1} ; \hat{Y}_{0} \mid X_{2}, Q\right) \\
& R_{2}<I\left(X_{2} ; \hat{Y}_{0} \mid X_{1}, Q\right), \\
& \text { subject to the constraints } \\
& H\left(\hat{Y}_{0} \mid X_{1}, Q\right)-H\left(\hat{Y}_{0} \mid Y_{0}\right)<I\left(X_{0} ; Y_{1}\right) \\
& H\left(\hat{Y}_{0} \mid X_{2}, Q\right)-H\left(\hat{Y}_{0} \mid Y_{0}\right)<I\left(X_{0} ; Y_{2}\right),
\end{aligned}
$$

for some $p\left(x_{0}, y_{1}, y_{2}\right)=p\left(x_{0}\right) p^{*}\left(y_{1}, y_{2} \mid x_{0}\right)$ and $p\left(q, x_{1}, x_{2}, \hat{y}_{0}, y_{0}\right)=p(q) p\left(x_{1} \mid q\right) p\left(x_{2} \mid q\right) p\left(\hat{y}_{0} \mid y_{0}\right) p^{*}\left(y_{0} \mid x_{1}, x_{2}\right)$ with the cardinality of $Q$ bounded as $|\mathcal{Q}| \leq 4$ and that for $\hat{Y}_{0}$ bounded as $\left.\left|\hat{\mathcal{Y}}_{0}\right| \leq|\mathcal{Y}|+3\right\}$.

Denote the closure of the set $\mathcal{R}$ by $\overline{\mathcal{R}}$. Since $\mathcal{C}$ is closed,

$$
\mathcal{R}_{\mathrm{CF}} \subseteq \mathcal{C} \Rightarrow \overline{\mathcal{R}_{\mathrm{CF}}} \subseteq \overline{\mathcal{C}}=\mathcal{C}
$$

The CF achievable region is derived by Schnurr et al. [8] for the half-duplex TWRC. The results can be readily extended to the full-duplex TWRC considered in this paper by setting $\alpha=1$ and $\beta=1$. The inequalities in (6) and (7) are strict due to the slight difference in the definition of achievable rate pairs in this paper and that in [8].

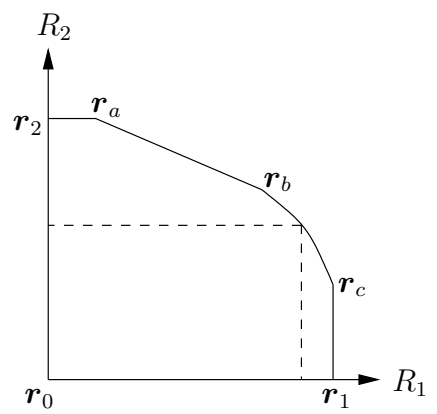

Fig. 2. The region of $\operatorname{Conv}\left(\mathcal{R}_{1}\right)$, which is the convex hull of $\mathcal{R}\left(X_{1}, X_{2}\right)$ for all $\left(X_{1}, X_{2}\right) \sim p\left(x_{1}\right) p\left(x_{2}\right)$. The dotted rectangle is an example of $\mathcal{R}\left(X_{1}^{\prime}, X_{2}^{\prime}\right)$ for some $p^{\prime}\left(x_{1}\right) p^{\prime}\left(x_{2}\right)$.

\section{The CAPACITy OF THE TWRC With A DETERMINISTIC UPLINK}

If the uplink of the TWRC is deterministic, we have that

$$
p^{*}\left(y_{0} \mid x_{1}, x_{2}\right)= \begin{cases}1, & \text { if } y_{0}=f\left(x_{1}, x_{2}\right) \\ 0, & \text { otherwise, }\end{cases}
$$

for some deterministic function $f\left(x_{1}, x_{2}\right)$. For this channel, we have the following capacity result:

Theorem 1: The capacity region of any restricted deterministic-uplink arbitrary-downlink TWRC is $\mathcal{C}=\mathcal{R}_{\text {out }}$.

\section{A. Points in $\operatorname{Conv}\left(\mathcal{R}_{1}\right)$}

If the uplink is deterministic, we have $H\left(Y_{0} \mid X_{1}, X_{2}\right)=0$. So, the RHS of (1) and (2) simplify to $I\left(X_{1} ; Y_{0} \mid X_{2}\right)=$ $H\left(Y_{0} \mid X_{2}\right)-H\left(Y_{0} \mid X_{1}, X_{2}\right)=H\left(Y_{0} \mid X_{2}\right)$ and $I\left(X_{2} ; Y_{0} \mid X_{1}\right)=H\left(Y_{0} \mid X_{1}\right)$ respectively. Therefore, we can re-write $\mathcal{R}_{1}$ as

$$
\begin{array}{r}
\mathcal{R}_{1}=\left\{\left(R_{1}, R_{2}\right) \in \mathbb{R}_{+}^{2}: R_{1} \leq H\left(Y_{0} \mid X_{2}\right), R_{2} \leq H\left(Y_{0} \mid X_{1}\right),\right. \\
\text { for some } \left.p\left(x_{1}, x_{2}, y_{0}\right)=p\left(x_{1}\right) p\left(x_{2}\right) p^{*}\left(y_{0} \mid x_{1}, x_{2}\right)\right\} .
\end{array}
$$

Before proving Theorem 1, we establish the following:

Lemma 3: For some deterministic $p^{*}\left(y_{0} \mid x_{1}, x_{2}\right)$ as defined in (11), any point in $\operatorname{Conv}\left(\mathcal{R}_{1}\right)$ can be written as $\left(H\left(Y_{0} \mid X_{2}, Q\right), H\left(Y_{0} \mid X_{1}, Q\right)\right)$ for some $p\left(q, x_{1}, x_{2}, y_{0}\right)=$ $p(q) p\left(x_{1} \mid q\right) p\left(x_{2} \mid q\right) p^{*}\left(y_{0} \mid x_{1}, x_{2}\right)$, where $Q$ is an auxiliary random variable with cardinality $|\mathcal{Q}|=3$.

Proof of Lemma 3: The region of the form $\operatorname{Conv}\left(\mathcal{R}_{1}\right)$ is depicted in Fig. 2. Define the top-right boundary as the boundary segment from $\boldsymbol{r}_{2}$ to $\boldsymbol{r}_{1}$ (via $\boldsymbol{r}_{a}, \boldsymbol{r}_{b}$, and $\boldsymbol{r}_{c}$ ), excluding $\boldsymbol{r}_{2}$ and $\boldsymbol{r}_{1}$. For a convex set $\mathcal{R}$, we call any point $\boldsymbol{r} \in \mathcal{R}$ such that $\boldsymbol{r} \notin \operatorname{Conv}(\mathcal{R}) \backslash\{\boldsymbol{r}\}$ a vertex of $\mathcal{R}$. In other words, a vertex cannot be formed by taking a weighted average of other points in $\mathcal{R}$. For example, in Fig. $2, \boldsymbol{r}_{0}, \boldsymbol{r}_{1}, \boldsymbol{r}_{2}, \boldsymbol{r}_{a}$, $\boldsymbol{r}_{b}, \boldsymbol{r}_{c}$, and all points from $\boldsymbol{r}_{b}$ to $\boldsymbol{r}_{c}$ on the top-right boundary are the vertices.

Recall that $\operatorname{Conv}\left(\mathcal{R}_{1}\right)$ is the convex hull of the union of rectangular regions of the form $\mathcal{R}\left(X_{1}^{\prime}, X_{2}^{\prime}\right) \triangleq\left\{0 \leq R_{1} \leq\right.$ $\left.H\left(Y_{0}^{\prime} \mid X_{2}^{\prime}\right), 0 \leq R_{2} \leq H\left(Y_{0}^{\prime} \mid X_{1}^{\prime}\right)\right\}$ for all $\left(X_{1}^{\prime}, X_{2}^{\prime}\right) \sim$ $p^{\prime}\left(x_{1}\right) p^{\prime}\left(x_{2}\right)$. So, any vertex $\boldsymbol{r}$ on the top-right boundary of $\operatorname{Conv}\left(\mathcal{R}_{1}\right)$ must be a vertex of some rectangle $\mathcal{R}\left(X_{1}, X_{2}\right)$ [i.e., $\left(H\left(Y_{0} \mid X_{2}\right), H\left(Y_{0} \mid X_{1}\right)\right)$ ], because $\boldsymbol{r}$ cannot be written as a weighted average of other points in 
$\operatorname{Conv}\left(\bigcup_{X_{1}, X_{2}} \mathcal{R}\left(X_{1}, X_{2}\right)\right) \backslash \boldsymbol{r}$. So, each of them must be $\left(H\left(Y_{0}^{\prime} \mid X_{2}^{\prime}\right), H\left(Y_{0}^{\prime} \mid X_{1}^{\prime}\right)\right)$ for some $p^{\prime}\left(x_{1}\right) p^{\prime}\left(x_{2}\right)$.

We now further show that $\boldsymbol{r}_{0}, \boldsymbol{r}_{1}$, and $\boldsymbol{r}_{2}$ can each be written as $\left(H\left(Y_{0} \mid X_{2}\right), H\left(Y_{0} \mid X_{1}\right)\right)$ for some $p\left(x_{1}\right) p\left(x_{2}\right)$. First, the largest value for $R_{1}$ in $\operatorname{Conv}\left(\mathcal{R}_{1}\right)$ cannot exceed

$$
\begin{aligned}
r_{1}^{\max } & =\max _{p\left(x_{1}\right) p\left(x_{2}\right)} H\left(Y_{0} \mid X_{2}\right) \\
& =\max _{p\left(x_{1}\right) p\left(x_{2}\right)} \sum_{x_{2}} p\left(x_{2}\right) H\left(Y_{0} \mid X_{2}=x_{2}\right) \\
& =\max _{x_{2}, p\left(x_{1}\right)} H\left(Y_{0} \mid X_{2}=x_{2}\right) .
\end{aligned}
$$

Let $X_{1}^{\prime} \sim p^{\prime}\left(x_{1}\right)$ and $X_{2}^{\prime}=x_{2}^{\prime}$ attain $r_{1}^{\max }$. Using this choice of input distribution, we have $H\left(Y_{0}^{\prime} \mid X_{1}^{\prime}\right)=0$. So, $\left(r_{1}^{\max }, 0\right)=\left(H\left(Y_{0}^{\prime} \mid X_{2}^{\prime}\right), H\left(Y_{0}^{\prime} \mid X_{1}^{\prime}\right)\right) \in \operatorname{Conv}\left(\mathcal{R}_{1}\right)$. Since the region $\operatorname{Conv}\left(\mathcal{R}_{1}\right)$ is convex, $\boldsymbol{r}_{1}$ must attain the largest value of $R_{1}$ in $\operatorname{Conv}\left(\mathcal{R}_{1}\right)$. Therefore, $\boldsymbol{r}_{1}=\left(H\left(Y_{0}^{\prime} \mid X_{2}^{\prime}\right), H\left(Y_{0}^{\prime} \mid X_{1}^{\prime}\right)\right)$. Similarly, by swapping the role of $X_{1}$ and $X_{2}$, we can show that $\boldsymbol{r}_{2}$ can be written as $\left(H\left(Y_{0}^{\prime \prime} \mid X_{2}^{\prime \prime}\right)=0, H\left(Y_{0}^{\prime \prime} \mid X_{1}^{\prime \prime}\right)\right)$ for some $X_{1}^{\prime \prime}=x_{1}^{\prime \prime}$ and $X_{2}^{\prime \prime} \sim p^{\prime \prime}\left(x_{2}\right)$. Fixing $X_{1}^{\prime \prime \prime}=x_{1}^{\prime \prime \prime}$ and $X_{2}^{\prime \prime \prime}=x_{2}^{\prime \prime \prime}, \boldsymbol{r}_{0}=\left(H\left(Y_{0}^{\prime \prime \prime} \mid X_{2}^{\prime \prime \prime}=x_{2}^{\prime \prime \prime}\right), H\left(Y_{0}^{\prime \prime \prime} \mid X_{1}^{\prime \prime}=x_{1}^{\prime \prime \prime}\right)\right)$.

We have shown that all vertices in $\operatorname{Conv}\left(\mathcal{R}_{1}\right)$ can be written as $\left(H\left(Y_{0} \mid X_{2}\right), H\left(Y_{0} \mid X_{1}\right)\right)$ for some $p\left(x_{1}\right) p\left(x_{2}\right)$. Since $\operatorname{Conv}\left(\mathcal{R}_{1}\right)$ is a closed convex set in a two-dimensional space, any point on its boundary can be written as a weighted average of two vertices. From Fig. 2, we see that any interior point in $\operatorname{Conv}\left(\mathcal{R}_{1}\right)$ can be written as a weighted average of a boundary point and $\boldsymbol{r}_{0}$. Hence, any point $\left(r_{1}, r_{2}\right) \in \operatorname{Conv}\left(\mathcal{R}_{1}\right)$ can be written as the weighted average of three vertices (the last one being $\boldsymbol{r}_{0}$ ), i.e.,

$$
\begin{aligned}
r_{1} & =\sum_{q \in\{a, b, c\}} p(q) H\left(Y_{0} \mid X_{2}\right)_{p^{q}\left(x_{1}\right) p^{q}\left(x_{2}\right)} \\
& =\sum_{q} p(q) H\left(Y_{0} \mid X_{2}, Q=q\right) \\
& =H\left(Y_{0} \mid X_{2}, Q\right),
\end{aligned}
$$

and similarly,

$$
r_{2}=H\left(Y_{0} \mid X_{1}, Q\right),
$$

where (13b), (13c), and (14) are evaluated with some $p\left(q, x_{1}, x_{2}, y_{0}\right)=p(q) p\left(x_{1} \mid q\right) p\left(x_{2} \mid q\right) p^{*}\left(y_{0} \mid x_{1}, x_{2}\right)$, and $Q$ is an auxiliary (time-sharing) random variable with cardinality $|\mathcal{Q}|=3$.

\section{B. Proof of Theorem 1}

With Lemma 3, we now prove Theorem 1. First, define $\mathcal{R}_{2}^{-}$ to be $\mathcal{R}_{2}$ where (3) and (4) are strict inequalities. Since $\mathcal{R}_{2}$ is closed, $\overline{\mathcal{R}_{2}^{-}}=\mathcal{R}_{2}$. Defining $\mathcal{R}_{\text {out }}^{-} \triangleq \operatorname{Conv}\left(\mathcal{R}_{1}\right) \cap \mathcal{R}_{2}^{-}$, we have

$$
\overline{\mathcal{R}_{\text {out }}^{-}}=\overline{\operatorname{Conv}\left(\mathcal{R}_{1}\right) \cap \mathcal{R}_{2}^{-}}=\operatorname{Conv}\left(\mathcal{R}_{1}\right) \cap \overline{\mathcal{R}_{2}^{-}}=\mathcal{R}_{\text {out }} .
$$

Next, define $\mathcal{R}_{\mathrm{CF}}^{+}$as $\mathcal{R}_{\mathrm{CF}}$ where (6) and (7) are inequalities (not necessarily strict). For deterministic uplink and choosing $\hat{Y}_{0}=Y_{0}$, the RHS of (6) and (7) become $I\left(X_{1} ; \hat{Y}_{0} \mid X_{2}, Q\right)=H\left(Y_{0} \mid X_{2}, Q\right)-H\left(Y_{0} \mid X_{1}, X_{2}, Q\right)=$ $H\left(Y_{0} \mid X_{2}, Q\right)$ and $I\left(X_{2} ; \hat{Y}_{0} \mid X_{1}, Q\right)=H\left(Y_{0} \mid X_{1}, Q\right)$ respectively. So,

$$
\begin{aligned}
\mathcal{R}_{\mathrm{CF}}^{+}=\{ & \left(R_{1}, R_{2}\right) \in \mathbb{R}_{+}^{2}: \\
& R_{1} \leq H\left(Y_{0} \mid X_{2}, Q\right), R_{2} \leq H\left(Y_{0} \mid X_{1}, Q\right),
\end{aligned}
$$

subject to the constraints

$$
\begin{aligned}
& H\left(Y_{0} \mid X_{1}, Q\right)<I\left(X_{0} ; Y_{1}\right), \\
& H\left(Y_{0} \mid X_{2}, Q\right)<I\left(X_{0} ; Y_{2}\right),
\end{aligned}
$$

for some $p(q) p\left(x_{1} \mid q\right) p\left(x_{2} \mid q\right) p\left(\hat{y}_{0} \mid y_{0}\right) p^{*}\left(y_{0} \mid x_{1}, x_{2}\right)$,

$$
\left.p\left(x_{0}\right) p^{*}\left(y_{1}, y_{2} \mid x_{0}\right) \text {, and }|\mathcal{Q}| \leq 4\right\} \text {. }
$$

$\mathcal{R}_{\mathrm{CF}}^{+}$is chosen to include some limit points of $\mathcal{R}_{\mathrm{CF}}$. Due to constrains (17) and (18), $\mathcal{R}_{\mathrm{CF}}^{+}$might not include all limit points of $\mathcal{R}_{\mathrm{CF}}$. So, we have that $\overline{\mathcal{R}_{\mathrm{CF}}^{+}}=\overline{\mathcal{R}_{\mathrm{CF} .}}$.

Now, pick any point $\left(r_{1}, r_{2}\right) \in \mathcal{R}_{\text {out }}^{-}$. Since $\left(r_{1}, r_{2}\right) \in$ $\operatorname{Conv}\left(\mathcal{R}_{1}\right)$, using Lemma $3, r_{1}$ and $r_{2}$ can be written as $H\left(Y_{0} \mid X_{2}^{\prime}, Q^{\prime}\right)$ and $H\left(Y_{0} \mid X_{1}^{\prime}, Q^{\prime}\right)$ respectively, evaluated with some $p^{\prime}(q) p^{\prime}\left(x_{1} \mid q\right) p^{\prime}\left(x_{2} \mid q\right)$. Also, since $\left(r_{1}, r_{2}\right) \in \mathcal{R}_{2}^{-}$, we have that $r_{1}=H\left(Y_{0} \mid X_{2}^{\prime}, Q^{\prime}\right)<I\left(X_{0}^{\prime} ; Y_{2}\right)$ and $r_{2}=$ $H\left(Y_{0} \mid X_{1}^{\prime}, Q^{\prime}\right)<I\left(X_{0}^{\prime} ; Y_{1}\right)$ for some $p^{\prime}\left(x_{0}\right)$. Hence $\left(r_{1}, r_{2}\right) \in$ $\mathcal{R}_{\mathrm{CF}}^{+}$. This means $\mathcal{R}_{\text {out }}^{-} \subseteq \mathcal{R}_{\mathrm{CF}}^{+}$, which implies that $\overline{\mathcal{R}_{\text {out }}^{-}} \subseteq \overline{\mathcal{R}_{\mathrm{CF}}^{+}}$.

Now, $\mathcal{R}_{\text {out }}=\overline{\mathcal{R}_{\text {out }}^{-}} \subseteq \overline{\mathcal{R}_{\mathrm{CF}}^{+}}=\overline{\mathcal{R}_{\mathrm{CF}}}$. From Lemma 1 , we have $\mathcal{C} \subseteq \mathcal{R}_{\text {out }}$; from (10), we have $\overline{\mathcal{R}_{\mathrm{CF}}} \subseteq \mathcal{C}$. Hence, we have $\mathcal{C}=\mathcal{R}_{\text {out }}$. This proves Theorem 1 .

\section{A Simpler Proof For ACHIEVABILITY}

In the previous section, we set $\hat{Y}_{0}=Y_{0}$ for CF. This suggests that we do not actually need to invoke WynerZiv coding and binning in CF. Here, we present a simpler proof (achievability) for the capacity region. Consider $B$ blocks, each containing $n$ channel uses. The $\ell$-th block of the uplink transmission and the $(\ell+1)$-th block of the downlink transmissions are as follows, for all $\ell \in\{1,2, \ldots, B-1\}$ :

- Choose some $p\left(x_{0}\right), p\left(x_{1}\right)$, and $p\left(x_{2}\right)$.

- User $i$ generates $2^{n R_{i}}$ length- $n$ sequences of $\boldsymbol{x}_{i}$ with each element randomly and independently selected according to $p\left(x_{i}\right)$, for $i \in\{1,2\}$. It transmits $\boldsymbol{x}_{i}\left(w_{i}\right)$.

- The relay receives $\boldsymbol{y}_{0}$, which is a deterministic function of $w_{1}$ and $w_{2}$. For the chosen codebooks $\left\{\boldsymbol{x}_{1}\left(w_{1}\right)\right\}$ and $\left\{\boldsymbol{x}_{2}\left(w_{2}\right)\right\}$, there are at most $2^{n\left(R_{1}+R_{2}\right)}$ unique sequences of $\boldsymbol{y}_{0}$. Index them $\boldsymbol{y}_{0}\left(w_{0}\right)$, for $w_{0} \in\{1,2, \ldots, M\}$, where $M \leq 2^{n\left(R_{1}+R_{2}\right)}$.

- The relay randomly generates $M$ length- $n$ sequences of $x_{0}$ with each element randomly and independently selected according to $p\left(x_{0}\right)$. It transmits $\boldsymbol{x}_{0}\left(w_{0}\right)$.

- User 1 decodes $w_{0}$ from its received symbols $\boldsymbol{y}_{1}$ and its own message $w_{1}$. It can do so reliably ${ }^{4}$ if $R_{2}<$ $I\left(X_{0} ; Y_{1}\right)$. We present the proof in Appendix A.

- Using the same argument, user 2 can reliably decode $w_{0}$ if $R_{1}<I\left(X_{0} ; Y_{2}\right)$.

- From $w_{0}$, both users know $\boldsymbol{y}_{0}$ exactly. User 1 can recover $w_{2}$ reliably and user 2 can recover $w_{1}$ reliably if $R_{1}<$ $I\left(X_{1} ; Y_{0} \mid X_{2}\right)$ and $R_{2}<I\left(X_{2} ; Y_{0} \mid X_{1}\right)$. We present the proof in Appendix B.

\footnotetext{
${ }^{3} \overline{\mathcal{R}_{\mathrm{CF}}^{+}}=\overline{\mathcal{R}_{\mathrm{CF}}}$ does not hold in general if we change the strict inequalities in (8) and (9) to inequalities, i.e, relaxing the constraints, which might lead to the inclusion of additional regions $\left\{\left(R_{1}, R_{2}\right)\right\}$ specified by (6) and (7).

${ }^{4}$ With arbitrarily small error probability
} 
- Each user $i$ transmits $(B-1)$ messages, each of size $2^{n R_{i}}$, over $B n$ channel uses. If the users can reliably decode their requested messages, the rate $\left(\frac{R_{1}(B-1)}{B}, \frac{R_{1}(B-1)}{B}\right)$ is achievable. Choosing a sufficiently large $B$, this scheme achieves rates arbitrarily close to $\left(R_{1}, R_{2}\right)$.

So, this scheme achieves any rate pair $\left(R_{1}, R_{2}\right) \in \mathcal{R}_{4}$, where

$$
\begin{aligned}
\mathcal{R}_{4}=\{( & \left.R_{1}, R_{2}\right) \in \mathbb{R}_{+}^{2}: \\
& R_{1}<\min \left\{I\left(X_{1} ; Y_{0} \mid X_{2}\right), I\left(X_{0} ; Y_{2}\right)\right\} \\
& R_{2}<\min \left\{I\left(X_{2} ; Y_{0} \mid X_{1}\right), I\left(X_{0} ; Y_{1}\right)\right\} \\
& \text { for some } p\left(x_{1}, x_{2}, y_{0}\right)=p\left(x_{1}\right) p\left(x_{2}\right) p^{*}\left(y_{0} \mid x_{1}, x_{2}\right) \\
& \text { and } \left.p\left(x_{0}, y_{1}, y_{2}\right)=p\left(x_{0}\right) p^{*}\left(y_{1}, y_{2} \mid x_{0}\right)\right\} .
\end{aligned}
$$

Using time sharing, the region $\operatorname{Conv}\left(\mathcal{R}_{4}\right)$ is achievable. Without the Wyner-Ziv coding constraints (8) and (9), $\mathcal{R}_{4}$ is in the same form as $\mathcal{R}_{1} \cap \mathcal{R}_{2}$. We can show that $\overline{\operatorname{Conv}\left(\mathcal{R}_{4}\right)}=$ $\mathcal{R}_{\text {out }}=\mathcal{C}$.

Remark 2: One can also show that noisy network coding (NNC) [11], which generalizes CF without using Wyner-Ziv coding, with $\hat{Y}_{0}=Y_{0}$ also achieves $\operatorname{Conv}\left(\mathcal{R}_{4}\right)$. Using NNC, encoding is also performed over $B$ blocks (with $B$ being sufficiently large), but decoding at the users is performed only after the last transmission block. Hence, NNC incurs a larger transmission-to-decoding delay and involves a more complicated decoding scheme (simultaneous decoding over all $B$ blocks) compared to the coding scheme described here.

\section{The UnRestricted CASE}

For the unrestricted TWRC, the problem is hard even with deterministic links. Suppose the uplink is a multiplier channel $Y_{0}=X_{1} X_{2}$, where $X_{1}, X_{2} \in\{0,1\}$, and the downlink consists of two noiseless orthogonal channels: $X_{0}=\left(X_{0}^{a}, X_{0}^{b}\right)$, $Y_{1}=X_{0}^{a}, Y_{2}=X_{0}^{b}$, where $X_{0}^{a}, X_{0}^{b} \in\{0,1\}$. Without loss of optimality ${ }^{5}$, the relay transmits $X_{0}^{a}=X_{0}^{b}=Y_{0}$. With this, we convert the unrestricted TWRC to the (unrestricted) multiplying two-way channel [10, pg. 634] where the capacity remains unknown to date.

\section{APPENDIX A}

Define the following subset of indices of the relay's codewords $\boldsymbol{x}_{0}\left(w_{0}\right)$, which have a one-to-one mapping to its received symbols $\boldsymbol{y}_{0}\left(w_{0}\right)$ :

$$
\begin{aligned}
\mathcal{S}_{w_{1}}(a)= & \left\{w_{0} \in\{1,2, \ldots, M\}: \boldsymbol{y}_{0}\left(w_{0}\right)=f\left(\boldsymbol{x}_{1}(a), \boldsymbol{x}_{2}(k)\right),\right. \\
& \text { for some } \left.k \in\left\{1,2, \ldots, 2^{n R_{2}}\right\}\right\} .
\end{aligned}
$$

Fixing user 1's message $w_{1}=a, \mathcal{S}_{w_{1}}(a)$ is the set of indices $w_{0}$ for which the corresponding $\boldsymbol{y}_{0}\left(w_{0}\right)$ are possible uplink outputs when the inputs are $\boldsymbol{x}_{1}(a)$ and $\boldsymbol{x}_{2}(k)$ for some $k$. Clearly, $\left|\mathcal{S}_{w_{1}}(a)\right| \leq 2^{n R_{2}}$ for any $a \in\left\{1,2, \ldots, 2^{n R_{1}}\right\}$.

Assuming that user 1 sent $\boldsymbol{x}_{1}\left(w_{1}=a\right)$, and the relay sent $\boldsymbol{x}_{0}\left(w_{0}=b\right)$. Receiving $\boldsymbol{y}_{1}$, user 1 declares that $\hat{w}_{0}$ was sent by the relay if it can find a unique index $\hat{w}_{0} \in \mathcal{S}_{w_{1}}(a)$ where $\left(\boldsymbol{x}_{0}\left(\hat{w}_{0}\right), \boldsymbol{y}_{1}\right) \in \mathcal{A}_{\epsilon}^{(n)}\left(X_{0}, Y_{1}\right)$, where $\mathcal{A}_{\epsilon}^{(n)}\left(X_{0}, Y_{1}\right)$ is the set

\footnotetext{
${ }^{5}$ Since the downlink is noiseless, any processing that the relay could have done (e.g., decoding or quantizing) can also be done at the users.
}

of jointly typical sequences [12, pg. 195]. Define the following events that can lead to incorrect decoding of $w_{0}$ :

$$
\begin{aligned}
& E_{1}:\left(\boldsymbol{x}_{0}(b), \boldsymbol{y}_{1}\right) \notin \mathcal{A}_{\epsilon}^{(n)}\left(X_{0}, Y_{1}\right) \\
& E_{2}:\left(\boldsymbol{x}_{0}(c), \boldsymbol{y}_{1}\right) \in \mathcal{A}_{\epsilon}^{(n)}\left(X_{0}, Y_{1}\right), \text { for some } c \neq b .
\end{aligned}
$$

By definition, $b \in \mathcal{S}_{w_{1}}(a)$, and using the asymptotic equipartition property (AEP) [12, pg. 196], we have that $\operatorname{Pr}\left\{E_{1}\right\}<\epsilon$, and

$$
\begin{aligned}
& \operatorname{Pr}\left\{E_{2}\right\} \leq \sum_{c \in \mathcal{S}_{w_{1}}(a) \backslash\{b\}}\left(\boldsymbol{x}_{0}(c), \boldsymbol{y}_{1}\right) \in \mathcal{A}_{\epsilon}^{(n)}\left(X_{0}, Y_{1}\right) \\
& \leq \sum_{c \in \mathcal{S}_{w_{1}}(a) \backslash\{b\}} 2^{-n\left(I\left(X_{0} ; Y_{1}\right)-3 \epsilon\right)} \\
& \leq\left(2^{n R_{2}}-1\right) 2^{-n\left(I\left(X_{0} ; Y_{1}\right)-3 \epsilon\right)}<2^{n\left(R_{2}-I\left(X_{0} ; Y_{1}\right)+3 \epsilon\right)} \leq 2^{-n \epsilon},
\end{aligned}
$$

if $R_{2} \leq I\left(X_{0} ; Y_{1}\right)-4 \epsilon$. Here, (21a) is due to the union bound, and $(21 \mathrm{~b})$ is due to the AEP. Hence, if $R_{2}<I\left(X_{0} ; Y_{1}\right)$, we can choose $\epsilon$ and $n$ such that $\operatorname{Pr}\left\{\hat{w}_{0} \neq w_{0}\right\} \leq \operatorname{Pr}\left\{E_{1}\right\}+$ $\operatorname{Pr}\left\{E_{2}\right\}<\epsilon+2^{-n \epsilon} \triangleq \eta$, where $\eta$ is arbitrarily small.

\section{APPENDIX B}

We use the results of the two-way channel [10], where two nodes, say $A$ and $B$, exchange data through the channel $p^{*}\left(y_{A}, y_{B} \mid x_{A}, x_{B}\right)$. It has been shown that the nodes can reliably exchange data if node $A$ transmits at rate $R_{A}<I\left(X_{A} ; Y_{B} \mid X_{B}\right)$ and node $B$ transmits at rate $R_{B}<$ $I\left(X_{B} ; Y_{A} \mid X_{A}\right)$ for some $p\left(x_{A}\right) p\left(x_{B}\right)$ [10]. To apply this result to the TWRC, we set $R_{A}=R_{1}, R_{B}=R_{2}, X_{A}=X_{1}$, $X_{B}=X_{2}$, and $Y_{0}=Y_{A}=Y_{B}$. Knowing $Y_{0}=Y_{A}=Y_{B}$, user 1 can reliably decode $w_{2}$ and user 2 can reliably decode $w_{1}$ if $R_{1}<I\left(X_{1} ; Y_{0} \mid X_{2}\right)$ and $R_{2}<I\left(X_{2} ; Y_{0} \mid X_{1}\right)$.

\section{REFERENCES}

[1] Y. Wu, P. A. Chou, and S. Kung, "Information exchange in wireless networks with network coding and physical-layer broadcast," in Proc. Conf. Inf. Sci. Syst. (CISS), Baltimore, USA, Mar. 16-18 2005.

[2] W. Nam, S. Chung, and Y. H. Lee, "Capacity bounds for two-way relay channels," in Proc. Int. Zurich Semin. Commun. (IZS), Zurich, Switzerland, Mar. 12-14 2008, pp. 144-147.

[3] L. Ong, S. J. Johnson, and C. M. Kellett, "The capacity region of multiway relay channels over finite fields with full data exchange," IEEE Trans. Inf. Theory, vol. 57, no. 5, pp. 3016-3031, May 2011.

[4] A. S. Avestimehr, A. Sezgin, and D. N. C. Tse, "Capacity of the twoway relay channel within a constant gap," Eur. Trans. Telecomm., vol. 21, no. 4, pp. 363-374, June 2010.

[5] W. Nam, S. Chung, and Y. H. Lee, "Capacity of the Gaussian two-way relay channel to within $\frac{1}{2}$ bit," IEEE Trans. Inf. Theory, vol. 56, no. 11, pp. 5488-5494, Nov. 2010.

[6] T. M. Cover and A. A. El Gamal, "Capacity theorems for the relay channel," IEEE Trans. Inf. Theory, vol. IT-25, no. 5, pp. 572-584, Sept. 1979.

[7] B. Rankov and A. Wittneben, "Achievable rate regions for the two-way relay channel," in Proc. IEEE Int. Symp. Inf. Theory (ISIT), Seattle, USA, July 9-14 2006, pp. 1668-1672.

[8] C. Schnurr, T. J. Oechtering, and S. Stanczak, "Achievable rates for the restricted half-duplex two-way relay channel," in Proc. 41st Asilomar Conf. Signal Syst. Comput., Pacific Grove, USA, Nov. 4-7 2007, pp. 1468-1472.

[9] S. L. Fong and R. W. Yeung, "Feedback enlarges capacity region of two-way relay channel," in Proc. IEEE Int. Symp. on Inf. Theory (ISIT), St Petersburg, Russia, July 31-Aug. 5 2011, pp. 2248-2252.

[10] C. E. Shannon, "Two-way communication channels," in Proc. 4th Berkeley Symp. Math. Stat. Probab., vol. 1. Univ. California Press, 1961, pp. 611-644.

[11] S. H. Lim, Y. Kim, A. El Gamal, and S. Chung, "Noisy network coding," IEEE Trans. Inf. Theory, vol. 57, no. 5, pp. 3132-3152, May 2011.

[12] T. M. Cover and J. A. Thomas, Elements of Information Theory, 2nd ed. Wiley-Interscience, 2006. 